\title{
Industrieforum · Industrial Forum
}

Verhaltenstherapie 1999;9:63

\section{Seroxat hat keine Zulassung gegen Schüchternheit!}

Stellungnahme zum Presserummel um Seroxat «Seroxat hat keine Zulassung gegen Schüchternheit!» Dies teilt das U nternehmen SmithK line B eecham mit. A nlaß hierfür sind jüngste Veröffentlichungen, in denen Seroxat als L ifestyle-Pille gegen Schüchternheit dargestellt wird. $D$ as verschreibungspflichtige A rzneimittel Seroxat ist in G roßbritannien jetzt für die Behandlung der sozialen A ngststörung/ sozialen Phobie zugelassen. Bereits seit 1991 ist Seroxat in England auf dem M arkt. In D eutschland wurde es 1992 einge- führt. Seroxat ist ein A ntidepressivum und wird gegen ernsthafte psychische Störungen eingesetzt. Die zugelassenen Indikationsgebiete sind: D epressive E rkrankungen, Z wangsstörung und Panikstörung. Diese stehen oft im Zusammenhang mit sozialer A ngststörung/sozialer Phobie.

Meldungen, die die Behandlung mit Seroxat für Menschen mit Schüchternheit in Verbindung bringen, sind falsch! Bei sozialer A ngststörung handelt es sich um eine ernstzunehmende E rkrankung, die bei Nichtbehandlung erhebliche A uswirkung auf das Leben der Patienten hat. 